\title{
The effect of a high fat diet on the susceptibility to nematode infection in mice
}

\author{
E. Funjika ${ }^{1}$, J.J. Worthington ${ }^{2}$, R.K. Grencis ${ }^{1}$, D.J. Thornton ${ }^{1}$ and Wellcome Trust Centre for \\ Cell-Matrix Research \\ ${ }^{1}$ Faculty of Biology, Medicine and Health, University of Manchester, M13 9PT and ${ }^{2}$ Faculty of Health and Medicine, \\ University of Lancaster, LA1 $4 Y G$.
}

Gastrointestinal nematode infections comprising Necator, Ancylostoma, Ascaris and Trichuris affect around a quarter of the world's population. These infections often result in chronicity and associated morbidity that brings huge socio-economic loss. Increased rural-urban migration in developing countries has led to adoption of new diets with increased consumption of sugary and fatty foods ${ }^{(1)}$. Therefore people not only face the detrimental challenge of worm infections but also that of diet-related illnesses. We are employing the well-described murine model of nematode infection, Trichuris muris, to investigate the effect of high fat diet (HFD) on the immune-regulated protection against these common parasites.

We infected C57BL/6 mice with a low dose of T. muris eggs, known to produce a chronic, Th1 cytokine mediated response in normal chow fed mice ${ }^{(2,3)}$. Mice were fed on either normal chow $(12 \%$ energy from fat) or HFD (60\% energy from fat) for 12 weeks before oral infection with a single or, to mimic natural infection, 9 weekly repeated trickle low doses of $T$. muris eggs. Mice were observed for either 42 days (single dose group) or for 2 weeks after final infection (repeated dose group) before comparing intestinal worm burdens, serum antibody responses, colon mucosal inflammation and cytokine production. Statistical analysis on collected data was performed using the student's t test and Mann-Whitney post-test. Data presented as mean $+/-$ SEM $(n=5)$. Statistical significance was considered when $\mathrm{P}<0.05(*), \mathrm{P}<0.01(* *)$.

As expected, the HFD fed mice gained more weight in comparison to the normal chow fed mice (Fig. 1). Strikingly, these obese, HFD fed mice had significantly lower worm burdens after either a single or repeated trickle low dose infection as compared to normal chow fed controls (Fig. 2). In addition the HFD mice produced less of the Th1, chronicity driving, cytokine interferon- $\gamma$ as compared to normal chow mice after a single dose (Fig. 3) or repeated doses of infection (data not shown).

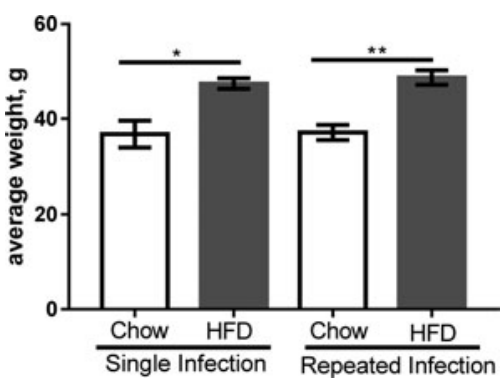

Fig. 1. Average mouse weight.

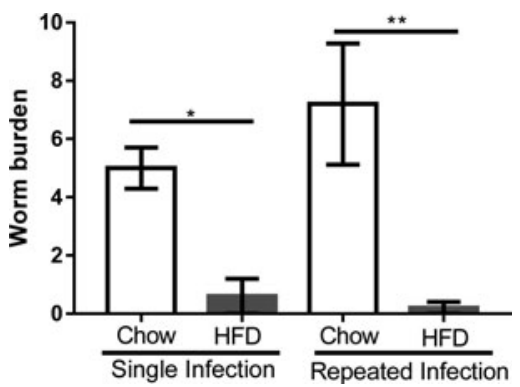

Fig. 2. Worm burden.

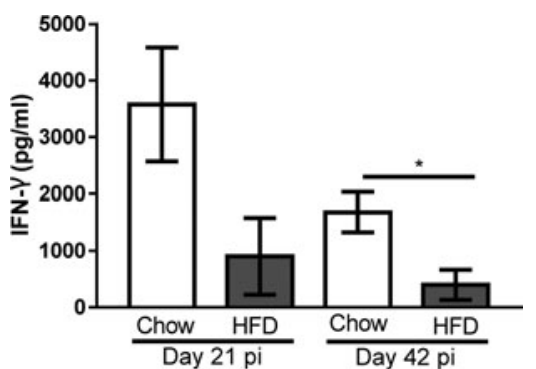

Fig. 3. Interferon- $\gamma$ production.

Our data suggests that dietary fat may influence resistance status to chronic intestinal nematode infection although the mechanism and how it can be used to boost immune responses in infected individuals remain to be clearly understood.

1. Caballero B (2005) NEJM 352, 1514-1516.

2. Bancroft AJ, McKenzie ANJ, Grencis RK (1998) J. Immunol 160, 3453-3461.

3. Hasnain SZ, Thornton DJ, Grencis RK (2011) Parasite Immunol 33, 45-5. 